Meta

Journal des traducteurs

Translators' Journal

\title{
Observations sur l'enrichissement lexical dans la progression vers un japonais « langue passive " pour l'interprétation de conférence
}

\section{Daniel Gile}

Volume 33, numéro 1, mars 1988

Traduction et interprétation au Japon

Translation and Interpretation in Japan

URI : https://id.erudit.org/iderudit/002915ar

DOI : https://doi.org/10.7202/002915ar

Aller au sommaire du numéro

Éditeur(s)

Les Presses de l'Université de Montréal

ISSN

0026-0452 (imprimé)

1492-1421 (numérique)

Découvrir la revue

Citer cet article

Gile, D. (1988). Observations sur l'enrichissement lexical dans la progression vers un japonais « langue passive " pour l'interprétation de conférence. Meta, 33(1), 79-89. https://doi.org/10.7202/002915ar

\section{Résumé de l'article}

Few Westerners have sufficient proficiency in Japanese for conference interpretation. The major stumbling block in their acquisition of Japanese as a passive language resides in vocabulary enhancement.Japanese vocabulary consists o/wago, kango and gairaigo and their compounds. Each category has different characteristics in terms of learning. While learning gairaigo is rather easy for the Westerner, wago proves more difficult to memorize, and kango poses special problems due to the small number of distinct syllables in Japanese as opposed to the large number of kanji used.

The large number of words used in Japanese compounds the difficulty, especially as compared with the acquisition of a Western language where the large proportion of words having common Greco-Latin roots that can be recognized even at first sight, reduces the number of new words that actually have to be learned.

These facts provide one explanation for the difficulty Westerners have in reaching an adequate level of comprehension of Japanese for interpretation purposes. They also raise questions as to the soundness of the philosophy interpreters' schools and their methods in developing high-level linguistic skills. Up to now, this question has been dealt with on the basis of" common sense " and the instructors' personal experience. Data obtained through scientific research may significantly contribute to an improvement of the situation.
Ce document est protégé par la loi sur le droit d'auteur. L'utilisation des services d'Érudit (y compris la reproduction) est assujettie à sa politique d'utilisation que vous pouvez consulter en ligne.

https://apropos.erudit.org/fr/usagers/politique-dutilisation/ 


\section{OBSERVATIONS SUR \\ L'ENRICHISSEMENT LEXICAL DANS LA PROGRESSION VERS UN JAPONAIS "LANGUE PASSIVE" POUR L'INTERPRÉTATION DE CONFÉRENCE*}

DANIEL GILE

INALCO, Paris, France

SU̧MMARY

Few Westerners have sufficient proficiency in Japanese for conference interpretation. The major stumbling block in their acquisition of Japanese as a passive language resides in vocabulary enhancement.

Japanese vocabulary consists of wago, kango and gairaigo and their compounds. Each category has different characteristics in terms of learning. While learning gairaigo is rather easy for the Westerner, wago proves more difficult to memorize, and kango poses special problems due to the small number of distinct syllables in Japanese as opposed to the large number of kanji used.

The large number of words used in Japanese compounds the difficulty, especially as compared with the acquisition of a Western language where the large proportion of words having common Greco-Latin roots that can be recognized even at first sight, reduces the number of new words that actually have to be learned.

These facts provide one explanation for the difficulty Westerners have in reaching an adequate level of comprehension of Japanese for interpretation purposes. They also raise questions as to the soundness of the philosophy interpreters' schools and their methods in developing high-level linguistic skills. Up to now, this question has been dealt with on the basis of "common sense " and the instructors' personal experience. Data obtained through scientific research may significantly contribute to an improvement of the situation.

\section{UN CONSTAT : LA RARETÉ DES INTERPRÈTES NON JAPONAIS TRAVAILLANT À PARTIR DU JAPONAIS}

Dans l'annuaire de l'AIIC (Association internationale des interprètes de conférence) daté du premier semestre 1986 figurent 21 interprètes ayant le japonais dans leur combinaison linguistique. Trois d'entre eux seulement sont d'origine non japonaise, et tous les trois ont un japonais " $C$ " (langue "passive " vers laquelle on ne travaille pas, mais qui peut servir de langue de départ). Hors de l'AIIC et notamment au Japon, sur plus de cent interprètes de conférence, les Occidentaux ayant le japonais dans leur combinaison linguistique professionnelle se comptent sur les doigts de la main.

Pourtant, à la différence d'autres marchés asiatiques, le marché japonais est vigoureux, et les interprètes de conférence y dépassent souvent 200 journées de travail par an, soit un chiffre supérieur à la moyenne pour les interprètes indépendants en Europe. En outre, contrairement à la Chine, le Japon est ouvert aux étrangers depuis la Deuxième Guerre mondiale, c'est-à-dire depuis que la profession d'interprète de confé- 
rence existe sous une forme " institutionnalisée ", et nombreux sont les Occidentaux qui y ont fait de longs séjours. Enfin, l'intérêt manifesté en Occident à l'égard du Japon ne cesse de croître depuis plusieurs années, ce qui se traduit notamment par une forte augmentation des effectifs des étudiants apprenant le japonais dans les universités occidentales. Au Département Corée-Japon de l'Institut national des langues et civilisation orientales, le nombre d'inscrits est passé de 41 personnes en 1960 à 1560 personnes en 1983 (Origas 1985 : 92), soit une augmentation de $300 \%$ environ. Le " cours d'initiation aux techniques de l'interprétation » comptait une trentaine d'étudiants en 1979 et plus de quatre-vingts en 1984. La première promotion du "cours d'initiation à la traduction scientifique et technique ", créé en 1979, comptait moins de dix étudiants ; en 1984, plus de trente y assistaient avec assiduité. Et pourtant, ce cours ardu et exigeant n'est pas obligatoire. Une telle augmentation des effectifs témoigne d'un intérêt croissant des étudiants non seulement vis-à-vis du japonais, mais aussi à l'égard des métiers de la traduction, que beaucoup envisagent d'embrasser avec la maîtrise du japonais qu'ils comptent acquérir à l'INALCO.

L'on constate toutefois que si en matière de traduction et d'interprétation de liaison, certains étudiants ont pu parvenir à un niveau opérationnel, surtout après un séjour de un ou deux ans au Japon, à une seule exception près, aucun n'est arrivé à une maîtrise suffisante du japonais pour l'interprétation de conférence. On notera d'ailleurs que même des étrangers résidant au Japon depuis dix ans et plus et vivant dans un milieu japonais n'arrivent pas à une compréhension " complète " de la langue au sens de l'AIIC (compréhension quasiment équivalente à celle d'un locuteur natif cultivé). S'ils ne se sentent nullement gênés dans la vie courante et s'expriment sans difficulté dans la vie professionnelle, même dans des domaines spécialisés tels que le droit, l'économie ou l'électronique, interrogés de manière précise, ils avouent en général ne pas tout comprendre dans le discours non spécialisé de registre moyen, tel que celui des émissions radiodiffusées et télévisées (Gile 1986b).

Â un niveau moins élevé, on constate qu'au regard de la compréhension du japonais oral, les étudiants de japonais ayant achevé leur cursus de licence ou de maîtrise tirent peu de profit d'un séjour de plusieurs mois ou un an au Japon, bien que leur capacité d'expression s'étoffe sensiblement à l'occasion de ce "bain linguistique ".

Ces observations contrastent avec celles que l'on peut faire à propos de l'apprentissage des langues occidentales : s'agissant des langues d'Europe de l'Ouest, un Occidental ayant acquis préalablement une base solide dans une langue étrangère peut s'attendre à parvenir à une compréhension quasiment "complète " de la langue après un séjour de quelques années dans le pays où elle est parlée ; on constate d'ailleurs que le niveau de compréhension du français, de l'anglais ou de l'allemand chez les Japonais ayant fait des séjours prolongés dans les pays concernés est en général sensiblement supérieur au niveau de compréhension du japonais des Occidentaux ayant fait des séjours linguistiques équivalents au Japon, bien que l'on ne puisse faire la même observation à propos de leur capacité d'expression.

Puisqu'il est question dans le présent article de la progression vers une langue de travail en interprétation de conférence, donc d'un perfectionnement poussé sur une base solide préalablement acquise, nous ne nous attarderons pas sur les difficultés grammaticales et syntaxiques, qui font partie du stade initial de l'apprentissage et que nous supposerons dépassées. Nous ne nous attarderons pas non plus sur une autre difficulté de la compréhension du japonais, qui tient à la nature elliptique, linguistiquement peu précise et " peu logique » du discours japonais, qui fait que dans cette langue bien plus que dans les langues occidentales, l'on peut avoir du mal à saisir le message d'un orateur tout en comprenant chacune de ses paroles (voir par exemple Mizutani 1981 ou Gile 1984a, 
1985b). Cet obstacle dans la compréhension est loin d'être insignifiant, comme en témoignent de nombreux Japonais, dont des interprètes de conférence qui se reportent à leur expérience professionnelle (voir, à titre illustratif, Doi 1974, Fukuii et Asano 1961 : 17-18, Kunihiro, Nishiyama et Kanayama 1968 : 52-53, Muramatsu 1974). Toutefois, si une démarche et des techniques précises peuvent être proposées pour lutter contre cette difficulté en traduction (voir Gile 1984a), il est difficile d'en faire autant en interprétation. En effet, si on dispose en traduction de plusieurs heures, voire de plusieurs jours pour résoudre les problèmes, en interprétation, des solutions doivent être trouvées en quelques minutes dans le mode de la consécutive et en quelques fractions de seconde quelques secondes au plus - dans le mode de la simultanée.

Or, ce problème ne prend tout son relief qu'une fois les obstacles proprement linguistiques écartés. Il apparaît cependant que les candidats à un japonais langue passive en interprétation butent sur un problème linguistique, plus précisément sur la difficulté de reconnaître une proportion non négligeable des mots dans le discours japonais.

C'est pourquoi nous nous concentrons dans les pages suivantes sur les aspects quantitatifs et qualitatifs du vocabulaire japonais qui constituent un obstacle majeur pour les étrangers, et notamment les Occidentaux, dans leur progression vers une compréhension "complète " et spontanée du japonais.

Le " corpus"

Dans les pages suivantes, il sera fréquemment fait allusion au "corpus". Il s'agit de la liste d'environ 12000 mots nouveaux rencontrés et appris par l'auteur sur une période d'" autoperfectionnement » de douze mois.

Le point de départ est une base lexicale préalable de 5000 mots environ ; cette estimation a été obtenue par énumération des entrées connues dans trente pages échantillonnées de manière aléatoire dans un dictionnaire japonais, et par multiplication de leur moyenne par le nombre de pages du dictionnaire en question.

Par la suite, les mots non compris rencontrés lors de la lecture de journaux, revues, textes non littéraires et non techniques et de romans d'action de registre linguistique moyen ont été systématiquement portés sur la liste. Par ailleurs, des mots inconnus gênants pour la compréhension du discours ou ayant attiré notre attention pour d'autres raisons, rencontrés lors de conversations avec des Japonais et lors de l'écoute d'émissions radiodiffusées et télévisées, y ont également été inscrits.

Ce «-corpus » représente donc la progression chronologique d'un appreneur entre 5000 et 17000 mots environ dans un registre moyen non spécialisé. Il ne s'agit que d'une étude de cas correspondant à un cheminement individuel et notamment à un choix particulier de textes et de discours, ce qui appelle une grande prudence dans toute extrapolation. Toutefois, en l'absence de corpus similaires sur des échantillons plus importants, certaines de ses caractéristiques les plus marquées serviront de référence ici.

\section{LES TROIS COMPOSANTS DU VOCABULAIRE JAPONAIS}

Le vocabulaire japonais se compose essentiellement de trois types de mots et de leurs composés : les wago ou yamatokotoba, mots d'origine autochtone, les kango, mots composés de un ou plusieurs caractères chinois juxtaposés, les uns importés de Chine, et les autres créés au Japon, et les gairaigo, mots d'origine occidentale dans leur quasitotalité et anglophones dans leur grande majorité (plus de 80,8\%, par opposition à $5,6 \%$ de gairaigo d'origine française et 3,3\% de gairaigo d'origine allemande dans les revues japonaises au début des années 1960 - Hayashi 1982 : 72), qui ont été morphologiquement et phonétiquement adaptés et naturalisés japonais.

Les wago représentent un peu moins de $40 \%$ des entrées dans un dictionnaire moyen ; les kango sont plus nombreux et représentent un peu plus de $52 \%$ des entrées ; 
les gairaigo sont minoritaires, avec $8 \%$, et les mots composés ne constituent que $2 \%$ environ du lexique présenté dans un tel dictionnaire non spécialisé (Hayashi 1982:60).

En ce qui concerne l'équilibre entre wago et kango, ces chiffres correspondent dans l'ensemble aux proportions des mots dans le corpus sur l'ensemble de la période de douze mois. Les gairaigo, par contre, y sont très peu représentés ; en effet, la plupart des gairaigo n'ayant pas subi d'importantes modifications morphologiques, phonétiques ou sémantiques (jenesuto pour general strike, aji bira pour agitation bill) sont transparents pour l'appreneur occidental et ne sont donc pas inscrits sur les listes : les gairaigo beddo mekingu (bed making), mozaiku (mosaic) et hiroiku (heroic), rencontrés au fil des lectures, n'apparaissent pas dans le corpus, car ils n'ont pas été considérés comme des mots nouveaux.

Il est intéressant de noter que l'apprentissage de chacun de ces trois types de mots pose pour les Occidentaux des problèmes différents.

\section{Les gairaigo}

Les gairaigo se retrouvent dans le vocabulaire japonais sous deux formes fondamentales : la plus simple pour l'appreneur occidental est celle que revêtent les emprunts nombreux et " sauvages " d'ailleurs, de mots occidentaux prononcés et transcrits à la japonaise mais ayant gardé leur sens originel. Dans ce cas, pour peu qu'ils comportent plus de deux syllabes, ils sont aisément reconnus et compris. S'ils ne se composent que d'une ou deux syllabes, leur transformation phonétique peut les rendre plus difficiles à reconnaître (penchi pour "pince "), mais ils sont faciles à retenir grâce à l'association mentale que l'on établit avec les mots d'origine.

D'autres gairaigo ont été empruntés il y a relativement longtemps et ont subi des transformations morphologiques et sémantiques plus ou moins importantes les rendant plus difficilement reconnaissables, ou sont devenus de " faux amis » à la suite d'un processus de dérive sémantique ( $a b e k k u$, tiré du français " avec ", désigne un couple ; mania, dérivé de "maniac", signifie "passionné de").

Dans tous ces cas, une fois le mot identifié et le lien avec le mot d'origine établi dans l'esprit de l'appreneur, l'association facilite grandement la rétention des gairaigo : dans des tests faits sur le corpus à plusieurs semaines, voire plusieurs mois du moment de l'apprentissage initial, les gairaigo ont dans leur quasi-totalité été reconnus et compris.

Dans l'ensemble, les gairaigo ne présentent donc pas de difficultés majeures pour l'appreneur occidentall'.

\section{Les wago}

Dans l'enrichissement lexical en japonais, si les gairaigo sont faciles à apprendre en tant que dérivés de mots déjà connus dans une langue occidentale, les wago, mots d'origine japonaise, sont entièrement nouveaux, comme le sont par exemple les mots allemands d'origine germanique pour un appreneur français.

Un élément important distingue toutefois l'apprentissage des wago de l'apprentissage des mots dans d'autres langues : dans la grande majorité de celles-ci, il existe une ferme association entre son, graphie et sens. Or, si les wago sont généralement écrits en hiragana, c'est-à-dire phonétiquement, ils peuvent également être écrits en partie ou en totalité en kanji ("caractères chinois"), qui en indiquent le sens mais moins souvent la prononciation $^{2}$.

Dans le premier cas, il existe une association triangulaire prononciationgraphisme-sens, qui fait que chaque fois qu'un mot est lu ou écouté, il peut être consi- 
déré comme ayant été " répété ". Dans le second cas, toutefois, un wago peut être compris sans être prononcé, et la lecture n'équivaut pas nécessairement à une répétition ${ }^{3}$.

Étant donné ces deux éléments, à savoir l'aspect graphique et la nature véritablemoent étrangère des wago pour l'appreneur, le taux de rétention dans cette catégorie quantitativement importante de mots japonais est très inférieur au taux de rétention des gairaigo. Dans le corpus, à quelques jours et quelques semaines de distance du moment de leur inscription, les wago n'ont été retenus que dans une proportion de l'ordre de $30 \%{ }^{4}$, et de nombreux wago " inconnus » rencontrés dans des textes ou discours se sont avérés avoir déjà été rencontrés et inscrits sur les listes.

\section{Les kango}

Dans le vocabulaire japonais, sur le plan de l'apprentissage comme sur celui de la perception du discours, les kango sont les éléments les plus intéressants.

En ce qui concerne l'association graphie-phonie-sens, le couple graphie-sens est fortement soudé en raison de la nature sémantiquement explicite des idéogrammes. Par contre, chacun de ceux-ci pouvant avoir plusieurs prononciations ("lectures") possibles, ce couple n'est pas étroitement associé à la phonie dans l'esprit de l'appreneur, et le lecteur peut appréhender le sens des kango sans savoir les prononcer, ce qui d'ailleurs arrive même à des Japonais. Il en résulte que dans les kango plus encore que dans les wago écrits partiellement en kanji, la lecture n'équivaut pas à une répétition auditive.

Un autre élément de difficulté, particulièrement important dans la progression vers le japonais langue passive en interprétation, est le très grand nombre d'homonymes parmi les kango: plus de 35\% des entrées dans des dictionnaires moyens ont des homonymes (Hayashi 1982 : 132), et compte tenu de la proportion des entrées que représentent les gairaigo et des wago, parmi lesquels les homonymies sont rares, le taux d'homonymie des kango dépasse de loin $50 \%$. Les homonymies dans les kango sont dues au fait que les 3000 kanji employés en japonais n'ont que 300 lectures environ, le système phonologique japonais étant très limité.

Si le contexte et la connaissance de la situation permettent de réduire sensiblement l'ampleur des difficultés de compréhension dues à ces homonymies, le problème reste non négligeable, et il n'est pas rare de voir des Japonais s'interroger sur le sens d'un kango prononcé en cours de conversation et s'expliquer en dessinant les kanji sur la paume de la main (voir à propos des kango : Gile 1986c). ble.

Pour l'appreneur étranger, le problème des homonymies dans les kango est dou-

- Contrairement aux langues occidentales, où la compréhension des mots non grammaticaux, et notamment les substantifs (une grande proportion des substantifs japonais sont des kango), peut aider à comprendre la phrase dans son ensemble, en japonais, c'est la compréhension de l'ensemble de la phrase qui aide à comprendre les substantifs, importants sur le plan informationnel.

- Dans l'apprentissage, l'écoute d'un kango non compris n'a pas la même utilité potentielle que l'écoute d'un mot non compris dans une langue occidentale. En effet, dans une langue occidentale, la familiarisation de l'appreneur avec le son d'un mot inconnu constitue un renforcement mnésique direct du deuxième élément de l'étroite association sens-phonie-graphie qui constitue ce mot, ainsi qu'un renforcement indirect du troisième élément en raison de la correspondance phonie-graphie dans les systèmes d'écriture alphabétique. En japonais, par contre, la phonie d'un kango est partagée entre plusieurs sens et plusieurs représentations graphiques, et le renforcement mnésique résultant de la répétition est bien plus faible. 
Au-delà des problèmes d'homonymie et parallèlement à ceux-ci, la pauvreté du système phonologique japonais par rapport au grand nombre de kanji utilisés fait que l'identification à l'écoute des constituants morphologiques des kango est difficile, chaque syllabe et chaque combinaison de deux syllabes étant susceptible de correspondre à plusieurs morphèmes différents : à titre d'exemple, selon les kanji employés, tai peut signifier " grand ", " gros ", désigner la plateforme, la matrice, la ceinture, le sac, le corps, l'idée d'opposition, d'attente, de paresse, d'endurance, de retrait, de substitution, de location, de change, de séjour, de troupe, et koosei indique la justice, l'offensive, la révision, la réhabilitation, le bien-être, les générations à venir, la lecture d'épreuves, l'organisation, etc.

Le phénomène est noté et souligné par les Japonais, notamment les interprètes interrogés par questionnaire sur les difficultés de la reconnaissance des kango (Gile 1986 c). A fortiori, il concerne les appreneurs étrangers, qui ne peuvent utiliser en japonais la connaissance des morphèmes pour analyser des mots nouveaux comme ils le peuvent dans des langues occidentales 5 . Ce sont d'ailleurs les kango qui ont été désignés comme présentant la principale difficulté de compréhension du japonais à l'écoute par une dizaine d'étrangers réputés comme ayant une excellente connaissance du japonais, dont trois interprètes travaillant à partir du japonais, que nous avons pu interroger longuement.

L'apprentissage des kango dans une optique de reconnaissance à l'écoute présente donc des problèmes particulièrement ardus pour l'interprète souhaitant acquérir le japonais comme langue passive professionnelle.

Par contre, quand ils sont écrits en kanji, les kango sont souvent compris par l'appreneur dès la première rencontre grâce à la nature sémantiquement éloquente des idéogrammes mentionnés plus haut (voir Gile 1984a). Même quand ils ne sont pas compris initialement par l'analyse des kanji, une fois leur sens élucidé, leur rétention est facilitée par une association mentale entre ce sens et le sens des kanji dont ils sont constitués (dans le corpus, les taux de rétention étaient supérieurs à $70 \%$ à quelques semaines de l'apprentissage initial). C'est pourquoi au cours du perfectionnement linguistique on arrive bien plus rapidement à une bonne compréhension des textes écrits qu'à une compréhension équivalente de l'oral.

\section{ÉLÉMENTS QUANTITATIFS}

Les interprètes n'ont pas dans leur bagage lexical permanent l'ensemble des termes techniques qu'ils sont appelés à employer dans les conférences spécialisées, et un travail de préparation complémentaire est presque toujours nécessaire (Gile 1985c, 1986a). Nous faisons donc abstraction de ce vocabulaire très spécialisé et considérons le bagage lexical du " généraliste " cultivé qu'est l'interprète. Si ce bagage était quantitativement limité et se composait de 3000 à 5000 mots, par exemple, un apprentissage initial et les répétitions intervenant naturellement dans l'environnement linguistique japonais lui permettraient probablement de retenir assez vite l'ensemble des wago, kango et gairaigo en dépit des caractéristiques évoquées ci-dessus.

La première question qui se pose est donc de savoir quel est l'ampleur du vocabulaire qui doit être maîtrisé, puisque ce paramètre détermine en grande partie la durée de la période de perfectionnement requise.

Le bon sens veut qu'en dépit des différences pouvant exister entre les langues, le vocabulaire utilisé hors du langage de la poésie et de la "grande littérature " reste dans un même ordre de grandeur. Des statistiques personnelles réalisées sur dictionnaires ayant situé le vocabulaire passif non technique et moyennement technique de quelques Français diplômés d'université et exerçant une profession intellectuelle autour de 
35000 mots, il semblait raisonnable de penser que c'était également le but à viser pour l'interprétation à partir du japonais.

Or, en examinant le vocabulaire appris dans les cours et méthodes de langues étrangères, le japonais ne faisant pas exception, on constate qu'à chaque leçon s'ajoutent quelques dizaines de mots nouveaux, et que le vocabulaire appris à chaque "niveau " est limité à quelques milliers de mots, soit un chiffre total inférieur en général à dix mille mots pour l'ensemble des niveaux d'un cours de langue non spécialisé.

L'acquisition du complément de 25000 mots environ entre les 35000 mots supposés requis et les 10000 mots acquis demanderait une progression de 5000 mots par an sur 5 ans ou de 2500 mots par an sur 10 ans, soit environ 14 mots nouveaux par jour dans le premier cas et 7 dans le second cas, et ce 365 jours par an.

Des statistiques personnelles réalisées lors de séjours à l'étranger nous donnent à penser que la rapidité d'acquisition de mots nouveaux par la voie naturelle du bain d'immersion linguistique est en réalité bien plus faible quand le point de départ est déjà élevé, et se situe plutôt à une moyenne inférieure à un mot par jour. Quant à des efforts délibérés qui permettraient éventuellement d'arriver aux chiffres ci-dessus, ils exigent une discipline qui paraît difficile à entretenir sur une période aussi longue.

Compte tenu de la réalité d'une compréhension quasiment " complète " d'une langue occidentale étrangère par des Occidentaux après une période de perfectionnement de quelques années seulement, on est amené à formuler deux hypothèses explicatives.

1. Le nombre de mots passifs nécessaires à cette compréhension "complète " d'une langue étrangère est très inférieur à la moyenne de 35000 prise comme référence, et peut donc être atteint assez rapidement.

Cette hypothèse trouverait sa justification dans le gradient des fréquences d'utilisation des mots, les mots de rang de fréquence peu élevé apparaissant si rarement qu'ils ne posent pas de problèmes réels. Les études sur les fréquences de mots réalisées depuis la fin du siècle dernier, notamment par Kaeding (1897), Meier (1964), Thorndike et Lorge (1944) et Zipf (1949) montrent en effet que dans toutes les langues, un petit nombre de mots différents constituent la grande masse des mots effectivement employés, et un très grand nombre de mots différents ne sont utilisés que rarement. Dans un travail de Meier sur l'allemand (1964: 53), 30\% environ de la masse des textes analysés se composent des 30 mots les plus fréquents et $50 \%$ de ces textes se composent des 200 mots les plus fréquents, alors que 217000 mots du vocabulaire allemand y représentent moins de $4 \%$ de la masse.

À cet égard, le nombre total de mots nécessaires à une compréhension " complète " semble nettement plus élevé en japonais que dans les langues occidentales. On ne dispose malheureusement pas de statistiques suffisantes; les enquêtes réalisées portent sur les quelques milliers de mots les plus fréquents, et indiquent qu'à ce niveau fondamental, le vocabulaire japonais est sensiblement plus varié que les vocabulaires anglais et français (les mille mots français les plus fréquents représentent $85,8 \%$ environ du vocabulaire utilisé, alors que les mille mots les plus fréquents en japonais n'en représentent que 60,5\% - Nakano 1975). Toutefois, pour l'interprète, ces chiffres sur les mille mots les plus fréquents ne sont pas significatifs, car il faut que la fréquence d'apparition des mots inconnus tombe bien en deçà de 1 mot sur 100 (100 mots représentent moins d'une minute de parole) pour qu'ils ne présentent plus de problèmes et que la compréhension puisse être considérée comme "complète ». Par ailleurs, des enquêtes réalisées au Japon font état d'un vocabulaire passif de l'ordre de 45000 à 50000 mots en moyenne chez les jeunes Japonais de 20 ans, et de l'ordre de 80000 mots ou davantage chez l'adulte cultivé, ce qui donne à penser que le vocabulaire total devant être su pour une compréhension "complète" est effectivement plus important en japonais qu'en 
français (Nakano 1975). Plus pertinent que ces chiffres mais fondé sur un cas individuel, le corpus donne des indications concrètes. On y trouve par exemple un taux de mots nouveaux par page de texte lu qui ne baisse pas de manière significative entre 6000 et 15000 mots acquis et qui oscille autour de 5 mots par page de roman d'action, soit environ 2 mots nouveaux par 100 mots non grammaticaux ${ }^{6}$ (avec des sautes occasionnelles à 10 mots ou davantage), ainsi qu'une compréhension toujours très imparfaite des émissions radiodiffusées et télévisées à 17000 mots.

Il est vrai que cette compréhension incomplète est due non seulement à l'insuffisance du nombre de mots " connus ", mais aussi au fait, expliqué plus haut, qu'en japonais la compréhension des kango à la lecture n'implique pas leur identification à l'écoute. Pour reconnaître un kango, il ne suffit donc pas de l'avoir vu souvent et de le comprendre instantanément à la lecture ; encore faut-il l'avoir reconnu à l'écoute suffisamment de fois pour que cette reconnaissance s'opère très rapidement et avec une dépense d'énergie minime, car le temps et l'énergie sont précieux en interprétation, et notamment en simultanée, où ils sont très limités (voir Gile 1985d). C'est pourquoi, bien que significatifs, les éléments présentés ci-dessus pour appuyer l'hypothèse d'une différence appréciable entre le nombre de mots requis pour une compréhension auditive "complète" en japonais et le vocabulaire minimum permettant une telle compréhension dans les langues occidentales demandent à être étayés par des statistiques complémentaires.

2. Un nombre important de mots peuvent être compris sans avoir été appris préalablement.

- D'une part, des éléments phonologiques, graphiques, morphologiques et grammaticaux permettent au lecteur ou à l'auditeur de dériver le sens du mot nouveau à partir d'un mot qu'il connaît déjà ("coder", "décoder", " codable" peuvent être compris à partir de "code").

- D'autre part, des éléments morphologiques, graphiques ou phonologiques communs à la langue maternelle de l'appreneur et à la langue dans laquelle il se perfectionne lui permettent souvent de reconnaitre un mot nouveau, au risque parfois de tomber dans le piège du "faux ami ".

À cet égard, pour l'appreneur occidental, les différences sont grandes entre le perfectionnement en japonais et le perfectionnement dans une nouvelle langue occidentale.

En effet, comme il a été expliqué plus haut à propos des hononymies, la compréhension à l'écoute par analyse de la composition morphologique des mots est difficile, voire impossible dans un grand nombre de mots japonais, à la différence de la plupart des mots anglais, français ou allemands par exemple 7 . Par ailleurs, contrairement à ces langues européennes, où le vocabulaire du locuteur cultivé a souvent des racines grécolatines communes permettant la compréhension instantanée par référence au mot correspondant dans la langue maternelle de l'appreneur, en dehors des gairaigo, le vocabulaire japonais ne présente pas de similitudes phonologiques avec le vocabulaire de la langue maternelle de l'appreneur occidental, et ne permet donc pas une telle modalité de compréhension.

\section{RACCOURCIR LA LONGUE MARCHE?}

La longueur de la progression vers une compréhension " complète " du japonais qui s'observe sur le terrain peut donc s'expliquer en partie par les éléments qualitatifs et quantitatifs présentés ci-dessus.

Il convient de souligner l'importance de l'aspect quantitatif du problème, souvent négligé. Les statistiques manquent en effet sur le vocabulaire des appreneurs de niveau élevé comme le sont les candidats à l'interprétation de conférence à partir du japonais. Des données manquent aussi sur le vocabulaire nécessaire à une compréhension " com- 
plète " d'une langue au sens de l'AIIC. Pourtant, il serait intéressant de cerner ce vocabulaire à des fins pédagogiques. À l'heure actuelle, la majorité des écoles d'interprétation occidentales refusent le rôle d'écoles de langue ${ }^{8}$ et considèrent que les futures langues de travail des apprentis-interprètes doivent être sues à l'admission (Nilski 1967, Keiser 1970, Geleff 1971, Lederer 1975, Gravier 1978). Et pourtant, une importante proportion des échecs des étudiants est attribuable à une connaissance insuffisante de ces langues. Si les étudiants admis sont jugés susceptibles de se perfectionner pendant le cursus, ne devrait-on pas essayer de leur proposer une orientation pédagogique plus efficace?

À l'heure actuelle, cette orientation est limitée dans la majorité des écoles, où l'on se contente en général de conseiller la lecture et l'écoute, en renvoyant les étudiants vers des journaux et revues d'une part, et vers des émissions radiodiffusées et télévisées, voire des "séjours linguistiques", d'autre part.

La répétition des mots et structures intervenant dans ces discours authentiques relativement proches du registre des conférences consolide sans doute dans la mémoire des étudiants le vocabulaire et les constructions et tournures qui y reviennent souvent.

Toutefois, sachant que la quasi-totalité des mots inconnus de l'apprenti-interprète et nécessaires à son bagage linguistique ont une fréquence d'utilisation très faible, l'on peut se demander si le rendement de l'apprentissage " naturel " que constituent l'écoute d'émissions et la lecture de textes authentiques n'est pas très faible, les mots et tournures à apprendre dans l'optique du perfectionnement qui nous intéresse ici n'apparaissant que rarement, par opposition à une grande masse de mots et de tournures très bien connus. En conséquence, peut-être y a-t-il lieu d'utiliser en partie des discours et textes " artificiels » destinés à ce perfectionnement.

Par ailleurs, l'on sait peu de choses sur les rapports entre l'apprentissage visuel (par la lecture) et la reconnaissance à l'écoute. L'existence d'une grande différence entre les deux en japonais est manifeste, mais elle est peut-être importante dans d'autres langues également, auquel cas la préparation de discours enregistrés spéciaux pourrait être utile.

Le japonais et son étude présentent des caractéristiques très marquées qui n'apparaissent pas aussi clairement dans les principales langues de travail des interprètes de conférence. Ces traits particuliers sont susceptibles de bousculer quelque peu les idées reçues et de conduire à des interrogations plus précises sur d'autres cas d'espèce.

Dans le domaine du peifectionnement linguistique, il existe peu de travaux portant sur le niveau qui nous intéresse ici ; aussi l'orientation pédagogique que donnent les enseignants sensibilisés au problème, fondée sur leur propre expérience, est-elle parfois excellente sur le plan qualitatif (voir par exemple Déjean le Féal 1976 et Ilg 1978), mais elle ne tient pas compte d'éléments quantitatifs. À la lumière des faits mis en relief à propos du japonais, n'est-on pas amené à se poser des questions sur l'efficacité du "bain d'immersion linguistique " comme méthode de perfectionnement, de l'utilisation de matériel authentique parce que " plus naturel ", de la lecture comme principal médium de progression vers une langue passive en interprétation de conférence, ou encore sur l'opportunité du refus des écoles d'interprètes de s'occuper du perfectionnement linguistique des apprentis-interprètes.

Aussi est-on amené une fois de plus (voir également Gile 1983a, 1983b, 1984b, 1985a, 1985d) à souligner l'intérêt d'une véritable recherche scientifique sur cette question, comme d'ailleurs sur l'ensemble des questions touchant l'interprétation de conférence, recherche qui permettrait de perfectionner les méthodes et d'améliorer tant la formation des interprètes que la qualité de leur travail. 
Notes

1. Il n'en est pas de mème pour l'appreneur chinois qui, contrairement aux Occidentaux, a une facilité évidente dans l'apprentissage des kango mais que les gairaigo déconcertent.

2. Le japonais s'écrit en kanji (" caractères chinois " ou "idéogrammes") et en hiragana ou en katakana, deux syllabaires se recoupant entièrement et se composant chacun de signes représentant phonêtiquement des syllabes. L'emploi de l'un ou de l'autre des systèmes est régi en partie par des " règles du bon usage ", mais reste dans une grande mesure à la discrétion de chacun.

3. Nous nous appuyons en grande partie dans notre raisonnement sur l'importance du nombre de répétitions intervenant lors de l'apprentissage sur la rétention lexicale, sans toutefois entrer ici dans les détails techniques des modalités de ces répétitions (intervalles, forme active ou passive, visuelle ou andiovisuelle, etc.) ; ces considérations dépassent en effet le cadre de notre propos.

4. Les chiffres portant sur les taux de rétention dans le corpus sont donnés à titre indicatif, car ces taux varient en fonction de différents paramètres de l'apprentissage initial, et notamment le nombre des répétitions et les écarts entre elles, qui ont été assez diversifiés au cours de la période de constitution du corpus.

5. La brièveté de la plupart des kango, composés de deux ou trois kanji, accroît cette difficulté en raison du manque de redondance phonologique interne dans le mot, et a fortiori dans ses morphèmes, qui ne constituent souvent qu'une syllabe correspondant à la lecture d'un kanji : kiko, tsugo, kikan, skakai,jiten, shain, etc. Le problème est moins aigu dans les wago, qui sont en général plus longs : okikaeru, erabikata, machigai, yorokobi, otogibanashi.

6. Les mots grammaticaux étant les conjonctions, enclitiques, etc., par opposition aux substantifs, verbes, adjectifs et adverbes qui véhiculent l'essentiel de l'information.

7. L'examen des différentes correspondances possibles entre son et kanji ou son et sème est en effet susceptible d'être très long. On notera par contre que la lecture d'un kanji ne passe apparemment pas nécessairement par sa prononciation, même mentale (voir Kaiho 1984).

8. Tel n'est pas le cas des écoles japonaises, par exemple, où l'étude de l'interprétation est un prolongement possible de l'étude de l'anglais.

* L'auteur remercie la Fondation du Japon dont une bourse de recherche à Tokyo lui a permis de réaliser plusieurs projets dont le présent travail, ainsi que la International Christian University qui l'a accueilli pendant son séjour au Japon.

\section{BIBLIOGRAPHIE}

DÉJEAN LE FÉAL, Karla (1976) : "Le perfectionnement linguistique ", Études de linguistique appliquée, $\mathrm{n}^{\circ} 24$, octobre-décembre.

DOI, Takeo (1974) : "Some Psychological Themes in Japanese Human Relationships ", dans John C. Condon and Mitsuko Saito, Intercultural Encounters with Japan, Tokyo, The Simul Press.

FUKUII, Haruhiro and Tasuke ASANO (1961) : Eigotsuuyaku no jissai, Tokyo, Kenkyuusha.

GELEFF, P. (1971) : «De deux mots, il faut choisir ... le meilleur ", Meta, 26-1.

GILE, Daniel (1983a) : «Aspects méthodologiques de l'évaluation de la qualité du travail en interprétation simultanée ", Meta, 28-3.

GILE, Daniel (1983b) : « Des difficultés de langue en interprétation simultanée ", Traduire, no 117, octobre.

GILE, Daniel (1984a) : "L'enseignement de la traduction japonais-français : une formation à l'analyse ", communication présentée au Colloque du CEDEL sur la communication entre langues européennes et orientales, Montvillargenne, janvier.

GILE, Daniel (1984b) : "La recherche : pourquoi et comment ", Bulletin de l'AIIC, 12-2, septembre.

GILE, Daniel (1985a) : "Théorie, modélisation et recherche dans la formation aux métiers de la traduction ", Lebende Sprachen, 24-1.

GILE, Daniel (1985b) : " La logique du japonais et la traduction des textes non littéraires : une présentation du problème ", Babel, 31-2.

GILE, Daniel (1985c) : "Les termes techniques en interprétation simultanée ", Meta, 30-3.

GILE, Daniel (1985d) : "L'interprétation de conférence et la connaissance des langues : quelques réflexions ", Meta, 30-4.

GILE, Daniel (1986a) : "Le travail terminologique en interprétation de conférence ", Multilingua, 5-1.

GILE, Daniel (1986b) : "Language Status Questionnaire : Report on Questionnaire Results ", JAT Bulletin, $n^{\circ} 17$, August.

GILE, Daniel (1986c) : "La reconnaissance des kango dans la perception du discours japonais ", Lingua 70, pp. 171-189.

GRAVIER, Maurice (1978) : "Pédagogie de la traduction ", dans Theory and Practice of Translation, Nobel Symposium 39, Stockholm, edited by Lillebil Grähs, Gustav Karlin, Bertil, Malmberg, ed. Lang, Berne. HAYASHI, Okii (1982) Zusetsu Nihongo, Tokyo, Kadokawa.

KAEDING, F.W. (1977) : Häufigskeitwörterbuch der deutschen Sprache, Berlin, Mittler Sohn. 
K.AIHO, Hiroyuki (1984) : Kenji wo kagaku suru, Tokyo, Yuhikyakusensho.

MEIER, H. (1964) : Deutsche Sprachlinguistik, vol. I et II, Hildesheim, G. Olms.

MIZUTANI, Osamu (1981) : "The Spoken Language in Japanese Life", The Japan Times, Tokyo.

MURAMATSU, Masumi (1974) : "Symposium on Conference Interpreting and Problems of Translation ", dans John C. Condon and Mitsuko Saito, Intercultural Encounters with Japan, Tokyo, The Simul Press.

ORIGAS, J.-J. (1985) : "Les études japonaises en France ", dans Japanese Studies in Europe, Tokyo, The Japan Foundation.

THORNDIKE, E.L. \& I. LORGE (1944) : The Teacher's Word Book of 30000 Words, New York, Bureau of Publications, Teacher's College, Columbia University.

ZIPF, G.K. (1949) : Human Behavior and the Principle of Least Effort, Cambridge, Massachusets, AddisonWesley. 